\title{
Coexistence of erythema dyschromicum perstans and vitiligo: a case report and review of the literature
}

\author{
Funda Tamer ${ }^{1} \bowtie$
}

\begin{abstract}
Erythema dyschromicum perstans is a rare, chronic, pigmentary disorder with unknown etiology. It clinically presents with oval to round, gray, blue, or brown macules of various sizes. The etiology remains unknown; however, cobalt allergy, radio contrast media, intestinal parasites, human immunodeficiency virus, and hypothyroidism have been proposed as causative factors. In addition, vitiligo is characterized by depigmented macules and patches that are widely and symmetrically distributed. It has been suggested that autoimmune mechanisms play an important role in the etiopathogenesis of vitiligo. Physical and emotional stress can trigger vitiligo in genetically predisposed patients. However, coexistence of erythema dyschromicum perstans and vitiligo is extremely rare, and similar immune mechanisms have been implicated in the pathogenesis of these cutaneous pigmentary disorders.
\end{abstract}

Keywords: erythema dyschromicum perstans, vitiligo

Received: 26 August 2016 | Returned for modification: 4 November 2016 | Accepted: 17 November 2016

\section{Introduction}

Erythema dyschromicum perstans (EDP) is a rare, chronic, pigmentary disorder with unknown etiology (1). It clinically presents with oval to round, gray, blue, or brown macules of various sizes. Symmetrically distributed lesions usually appear on the face, neck, trunk, and extremities $(1,2)$. The etiology remains unknown; however, cobalt allergy, radio contrast media, intestinal parasites, human immunodeficiency virus, and hypothyroidism have been proposed as causative factors $(2,3)$. Moreover, it has been suggested that EDP is associated with lichen planus. Patients that have lichen planus and EDP together have been reported previously $(1,3)$. There is controversy whether EDP is a subtype of lichen planus or a distinct entity. Histopathological findings usually show perivascular lymphocytic infiltration, melanophages, vacuolization of the basal layer, and necrotic keratinocytes (4). The disease should be differentiated from lichen planus pigmentosus, postinflammatory hyperpigmentation, fixed drug eruption, and Addison's disease. Antibiotics, corticosteroids, antihistamines, dapsone, chloroquine, clofazimine, and isotretinoin are the treatment of choice. However, none of them provide an effective treatment (5).

Vitiligo is characterized by depigmented macules and patches that are widely and symmetrically distributed. Autoimmune mechanisms play an important role in the etiology. Physical and emotional stress can trigger vitiligo in genetically predisposed patients. Furthermore, oxidative stress can increase melanocyte destruction. Corticosteroids, calcineurin inhibitors, vitamin D analogues, oral vitamins, phototherapy, and laser therapy are the treatment options (6).

Coexistence of erythema dyschromicum perstans and vitiligo is extremely rare. However, similar immune mechanisms have been implicated in the pathogenesis of these cutaneous pigmentary disorders.

\section{Coexistence of erythema dyschromicum perstans and vitiligo}

A 23-year-old Caucasian male patient complaining of changes in skin color was admitted for further clinical evaluation. Dermatological examination revealed grayish, hyperpigmented, excoriated macules and plaques on the back and occipital region, and mild hyperpigmentation on the upper chest. In addition, there was a well-demarcated, oval, depigmented patch $10 \mathrm{~cm}$ in diameter on his left shoulder (Figs. 1a-b).

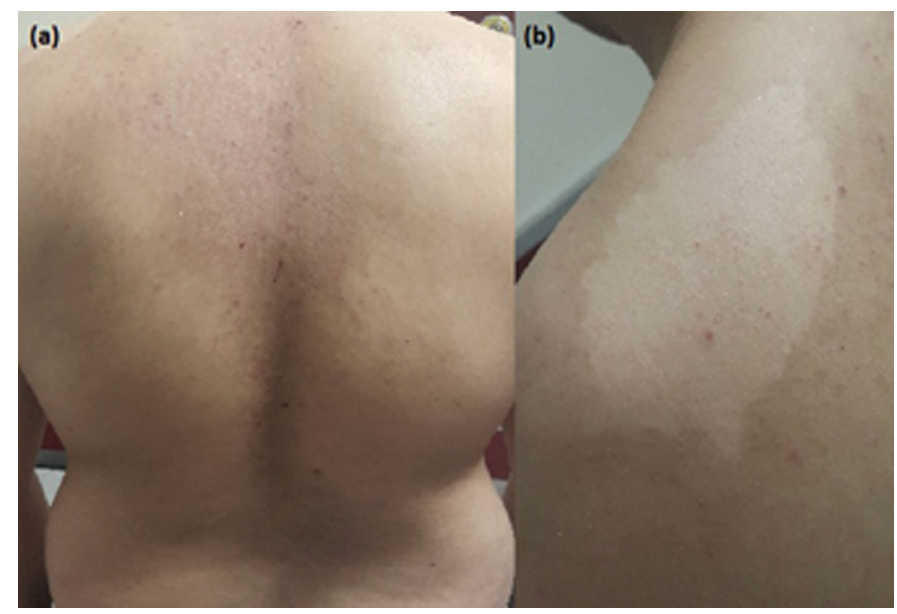

Figure 1 | a) Hyperpigmented, grayish, excoriated macules and plaques on the back; b) Oval depigmented patch on the left shoulder.

Wood's lamp examination showed discrete depigmentation with sharp borders. The patient admitted that the depigmented lesion had been present for the last 6 months. Moreover, the lesion appeared as a small macule and it gradually increased in size. It was asymptomatic and there were no other depigmented macules elsewhere. Thus, the diagnosis of vitiligo was made based on the clinical features and Wood's lamp examination. Furthermore, the patient stated that the hyperpigmented lesions first appeared on the back and had extended to the occipital region and chest over the last 5 years. He had used topical corticosteroids previously, but no clinical response had been achieved. The past medical history was unremarkable. He denied taking any medication. The skin biopsy was taken from the hyperpigmented lesions on the middle of the back. Histopathological examination revealed vacuolar degeneration on the basal layer, melanophages, and 
lymphocytic infiltration in the upper dermis. Periodic acid-Schiff, crystal violet, and Congo red stains were performed and the specimen did not show any amyloid deposits or metachromasia (Fig. 2). Therefore we confirmed the diagnosis of erythema dyschromicum perstans. The patient was put on topical steroid and oral antihistaminic treatment.

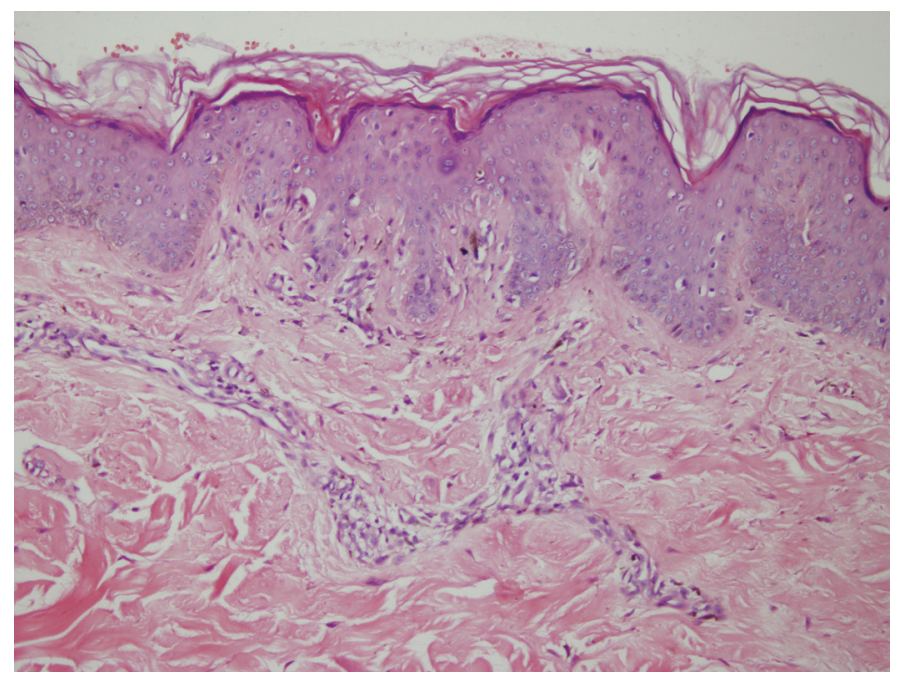

Figure 2 | Vacuolar degeneration on the basal layer, melanophages, and lymphocytic infiltration in the upper dermis $(H \& E \times 200)$.

The patient we presented above had both EDP and vitiligo at the same time. Only two similar cases have been reported previously. Henderson et al. presented a 31-year-old man that had EDP and developed depigmented patches on his elbows and knees in the previous year. The patient stated that the slowly progressive, hyperpigmented lesions had been present for a long time. The patient had cosmetic concerns and he did not have any other illnesses. Dermatological examination revealed gray macules with erythematous borders on the chest and proximal aspect of the arms and legs. In addition, there were depigmented macules with sharp borders on the knees, elbows, and distal site of the legs. The skin biopsies were performed from a hyperpigmented and a depigmented lesion. Histopathological examination showed an absence of melanin in the depigmented area. However, there was melanin in the upper dermis and basal area, vacuolization of the basal layer, and pigment incontinence in the hyperpigmented lesion. Therefore the diagnosis of EDP and vitiligo were both confirmed histopathologically (7).

Naik reported a 33-year-old man with a 6-month history of EDP and a 20-year history of depigmented patches on the trunk and extremities. The patient had been treated with light exposure 5 years previously. The patient revealed that he did not have any preceding lesions or hypoesthesia in the affected areas. The past medical history and family history were both unremarkable. Dermatological examination revealed scaly, gray-blue patches on the trunk and arms. Furthermore, there were depigmented patches on the dorsal site of the legs, hands, upper chest, back, and lips. Wood's lamp examination confirmed depigmentation, but hyperpigmented patches did not fluoresce under Wood's lamp. A skin biopsy was performed from the active border of a gray-blue patch. Histopathological examination revealed superficial, perivascular infiltration of lymphocytes and melanophages. However, the number of melanocytes in the basement membrane was normal. Therefore, the patient was diagnosed with EDP and vitiligo (1).

Gross et al. performed immunocytochemical analysis of leukocyte infiltrates in the affected skin of EDP and vitiligo patients. They showed similar subpopulations including $\mathrm{CD}_{3+}, \mathrm{CD} 8+, \mathrm{T}-$ suppressor, macrophages, and T-cytotoxic cells in the epidermis and Ia antigen positivity of the dendritic cells and lymphoid cells in the infiltrates of both diseases. Therefore, they suggest similar immune mechanisms in these cutaneous pigmentary disorders (8).

\section{Conclusion}

This article has reported an extremely rare case of EDP coexisting with vitiligo. To the best of our knowledge, no new cases have been reported since 2003. It has been considered that EDP may be a form of lichen planus because of the similar immunopathological features. However, these cases suggest that common immunological mechanisms may also be responsible for the coexistence of EDP and vitiligo. It should be considered that patients with EDP may develop other dermatological disorders, including lichen planus and vitiligo.

\section{References}

1. Naik NS. Erythema dyschromicum perstans and vitiligo. Dermatol Online J. 2003;9:25.

2. Tlougan BE, Gonzalez ME, Mandal RV, Kundu RV, Skopicki D. Erythema dyschromicum perstans. Dermatol Online J. 2010;16:17.

3. Schwartz RA. Erythema dyschromicum perstans: the continuing enigma of Cinderella or ashy dermatosis. Int J Dermatol. 2004;43:230-2.

4. Vásquez-Ochoa LA, Isaza-Guzmán DM, Orozco-Mora B, Restrepo-Molina R, Trujillo-Perez J, Tapia FJ. Immunopathologic study of erythema dyschromicum perstans (ashy dermatosis). Int J Dermatol. 2006;45:937-41.

5. Wang F, Zhao YK, Wang Z, Liu JH, Luo DQ. Erythema dyschromicum perstans response to isotretinoin. JAMA Dermatol. 2016;152:841-2.

6. Manga P, Elbuluk N, Orlow SJ. Recent advances in understanding vitiligo. F100oRes. 2016:6;5.

7. Henderson CD, Tschen JA, Schaefer DG. Simultaneously active lesions of vitiligo and erythema dyschromicum perstans. Arch Dermatol. 1988;124:1258-60.

8. Gross A, Tapia FJ, Mosca W, Perez RM, Briceno L, Henriquez JJ, et al. Mononuclear cell subpopulations and infiltrating lymphocytes in erythema dyschromicum perstans and vitiligo. Histol Histopathol. 1987;2:277-83. 